\title{
Polymerisation on Bio-Tissues
}

\author{
Hiromasa Goto* \\ Division of Materials Science, Faculty of Pure and Applied Sciences, \\ University of Tsukuba, Tsukuba, Ibaraki 305-8573, Japan \\ E-mail: gotoh@ims.tsukuba.ac.jp (H. Goto)
}

Keywords: bacterial electrolyte solution; PEDOT; polymerisation, polypyrrole, wood.

\begin{abstract}
Preparation of electro-active polymers having characteristic surface on biological tissue was carried out. Direct polymerisation on biological material with unique structure can be a new method to obtain functional structure with no use of top-down or bottom-up technologies. Polymerisations of pyrrole, aniline, and 3,4-ethylenedioxythiophene (EDOT) were carried out on the bio-tissues. Surface structure of the bio-tissue/conducting polymer composite was observed with optical microscopy. The results of the present study involve demonstration of deposition of conducting polymers on the surface of wood, membrane of egg, fungus, flower, and bacteria in the water medium. This method allows preparation of electro-active composites with ordered structure through combination of structures of biological tissues. Note that electrochemical polymerisation in bacterial electrolyte solution can be a first example to date.
\end{abstract}

\section{Introduction}

Conducting polymers have been paid attention from view points of application for solar cells [1,2], antistatic materials [3], and electroluminescent devices [4]. Bottom up and top down techniques have been employed for preparation of opto-electronic functional materials with ordered structure based on biomimetic technologies. Recently, we have carried out synthesis of polyaniline as a conducting polymer on biological materials to obtain certain organised forms with electro-activity $[5,6]$. Conducting polymers have possibility for construction of composite with functional materials. Bio-interface between nerve tissues/cells and advanced functional biocompatible polymers for neuroregeneration has been studied [7]. Polypyrrole can be prepared in the presence of other materials such as hydroxybenzoic acid [8].

Combination of bio-materials and $\pi$-conjugated polymers may be another option to obtain electro-active polymers having bio-organization like structure.

\section{Experiment}

\section{Bio-materials}

Bio-film (fungus), chamaecyparis obtuse (flower), shell membrane of egg, and chamaecyparis obtuse (wood) were used for bio-templates.

Polymerisation of aniline on the surface of Japanese cypress

Chamaecyparis obtuse (wood, $1.12 \mathrm{~g}$ ) was added into a solution of aniline $(0.5 \mathrm{~g})$ and sulfuric acid $(0.9 \mathrm{~g})$ in distilled water $(50 \mathrm{~mL})$, and stirred for $24 \mathrm{~h}$. Then, a solution of ammonium sulfate in $50 \mathrm{~mL}$ of distilled water was gradually added to the solution. The mixture was stirred for 2 days at ca. $0{ }^{\circ} \mathrm{C}$. The crude product was washed with large volume water for $30 \mathrm{~min}$, and methanol for $30 \mathrm{~min}$. The resultant composited was dried under reduced pressure. As prepared polyaniline (PANI) is a doped form with sulfuric acid (Scheme 1). Treatment with ammonia/water yields dedoped form of PANI. 


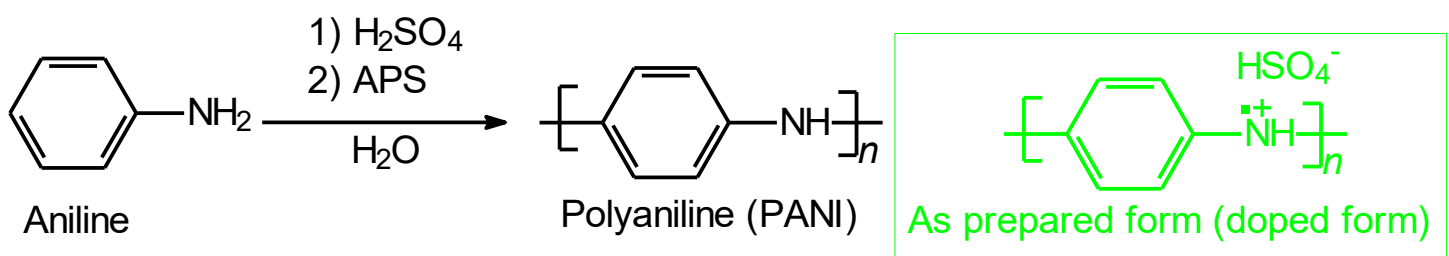

Scheme 1. Polymerisation of aniline.

Polymerisation of pyrrole

The bio-material (bio-film, Japanese camellia, or shell membrane of egg) was immersed into a solution containing small amount of $\mathrm{FeCl}_{3}$ in the water. After ca. 1h, the surface of the biomaterial was gently tapped with dry paper to absorb excess amount of the water solution. Subsequently, a solution of pyrrole in water was dropped onto the bio-tissue. In this case, pyrrole was partly dissolved in water. Polymerisation of pyrrole was carried out on the tissues surface containing $\mathrm{FeCl}_{3}$ as an oxidiser for polymerisation (Scheme 2). The colour of the surface was changed to black accompanied by deposition of polypyrrole on the surface. The resultant material was washed with methanol and dried. As prepared polypyrrole is a doped form having electrical conductivity. In this case, $\mathrm{FeCl}_{3}$ as a Lewis acid is a dopant for the polypyrrole after polymeisation. Quantity used: Membrane of egg $(1 \mathrm{~g})$, water $(10 \mathrm{~mL})$, pyrrole $(0.22 \mathrm{~g})$; Japanese camellia (flower) (1.33 g), pyrrole $(0.15 \mathrm{~g})$, water $(20 \mathrm{~mL})$.

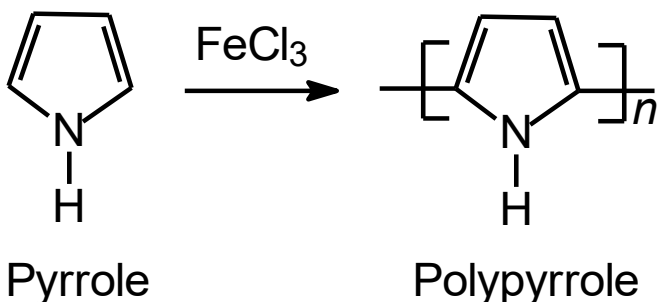

Scheme 2. Polymerisation of pyrrole.

Electrochemical polymerisation of 3,4-ethylenedioxythiophene (EDOT)

An image of solution of fermented soybeans bacteria solution $(5 \mathrm{~g})$ is shown in Fig. 1. A small amount of 3,4-ethylenedioxythiophene (EDOT, $0.2 \mathrm{~g}$ ) was added to a solution of fermented soybeans bacteria in the water. The solution containing monomer was electrochemically polymerised with application of voltage of $3.5 \mathrm{~V}$ (direct current, dc) (Scheme 3). After 30 min, resultant blue coloured film deposited on the anode $(+)$ side of the electrode (indium-tin-oxide coated glass, ITO) was washed with water, and methanol. The thin film deposited on ITO was dried.

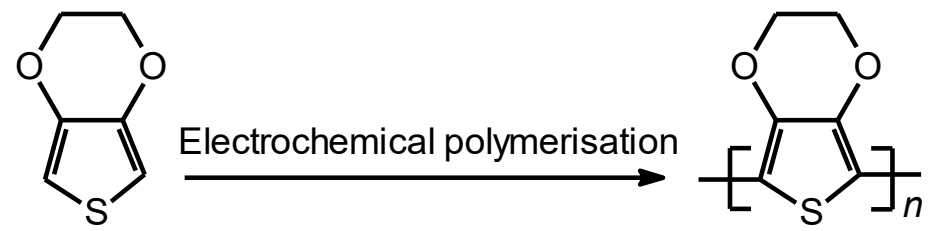

3,4-Ethylenedioxythiophene (EDOT)

Poly(3,4-ethylenedioxythiophene) (PEDOT)

Scheme 3. Polymerisation of 3,4-ethylenedioxythiophene (EDOT). 


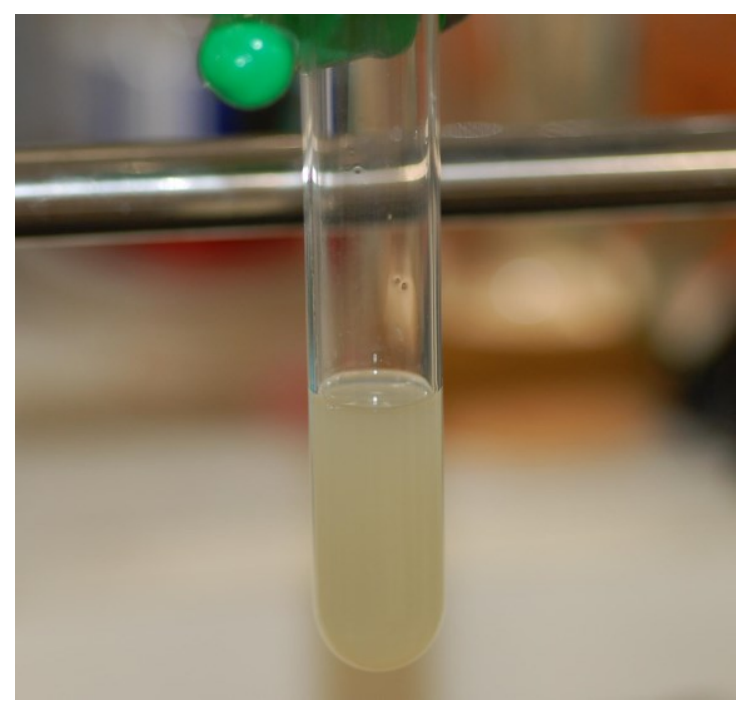

Figure 1. Fermented soybeans bacteria in the water.

Table 1. Preparation of conducting polymer deposited on bio-materials.

\begin{tabular}{lccccc}
\hline Bio-material & Monomer & Solvent & Acid & Oxidiser & Method \\
\hline Japanese cypress (wood) & Aniline & Water & $\mathrm{H}_{2} \mathrm{SO}_{4}$ & $\mathrm{APS}^{\mathrm{a}}$ & $\mathrm{Chem}^{\mathrm{b}}$ \\
Shell membrane & Pyrrole & Water & - & $\mathrm{FeCl}_{3}$ & $\mathrm{Chem}^{\mathrm{b}}$ \\
Bio-film (fungus) & Pyrrole & Water & - & $\mathrm{FeCl}_{3}$ & $\mathrm{Chem}^{\mathrm{b}}$ \\
Chamaecyparis obtuse (flower ) & Pyrrole & Water & - & $\mathrm{FeCl}_{3}$ & $\mathrm{Chem}^{\mathrm{b}}$ \\
Fermented soybeans bacteria & EDOT & Water & - & $-^{\mathrm{b}}$ & $\mathrm{EC}^{\mathrm{c}}$ \\
\hline
\end{tabular}

${ }^{\mathrm{a}}$ Ammonium persulfarte.

${ }^{\mathrm{b}}$ Chemical oxidative polymerisation.

${ }^{\mathrm{c}}$ Electrochemical oxidative polymerisation.

Surface observations

Fig. 2 shows surface structure of wood/polyaniline. Polyaniline was deposited on the cells of the wood. Polymerisation on the vessel of the wood allows highly ordered structure.

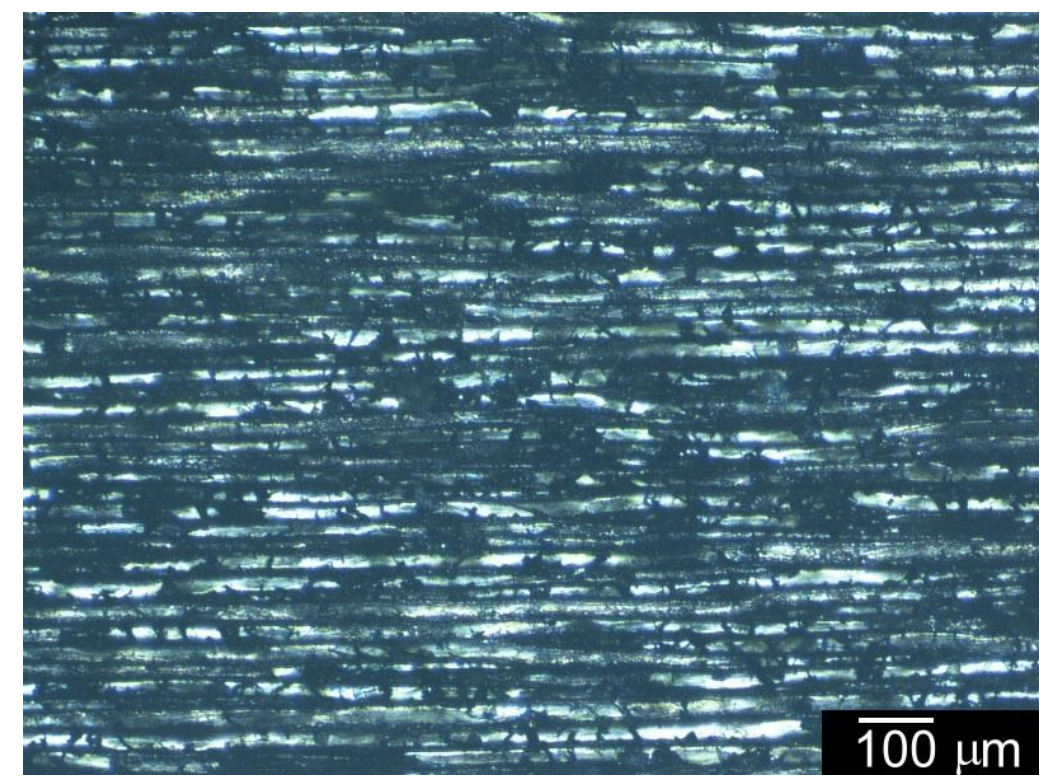

Figure 2. Optical microscopy image of polyaniline deposited on chamaecyparis obtuse with reflection light. 
Fig. 3 shows surface structure of bio-film/polypyrrole. Polypyrrole was deposited on the fungi. Micro-strings like structure were formed on the bio-film (fungi).

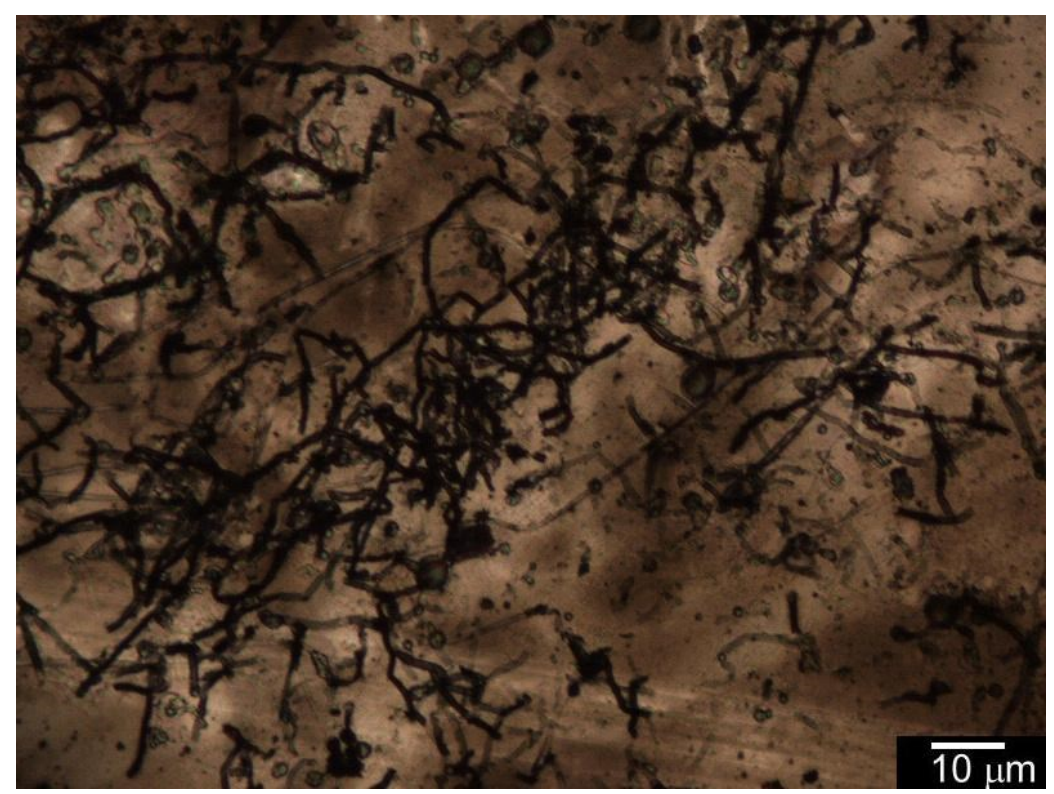

Figure 3. Optical microscopy image of polypyrrole deposited on chamaecyparis obtuse with reflection light.

Fig. 4 shows surface structure of Japanese camellia/polypyrrole. Polypyrrole can deposit on the soft tissue of the flower. Combination of polypyrrole and the flower allows formation of small cell structure as an organic conductor in micro-size.

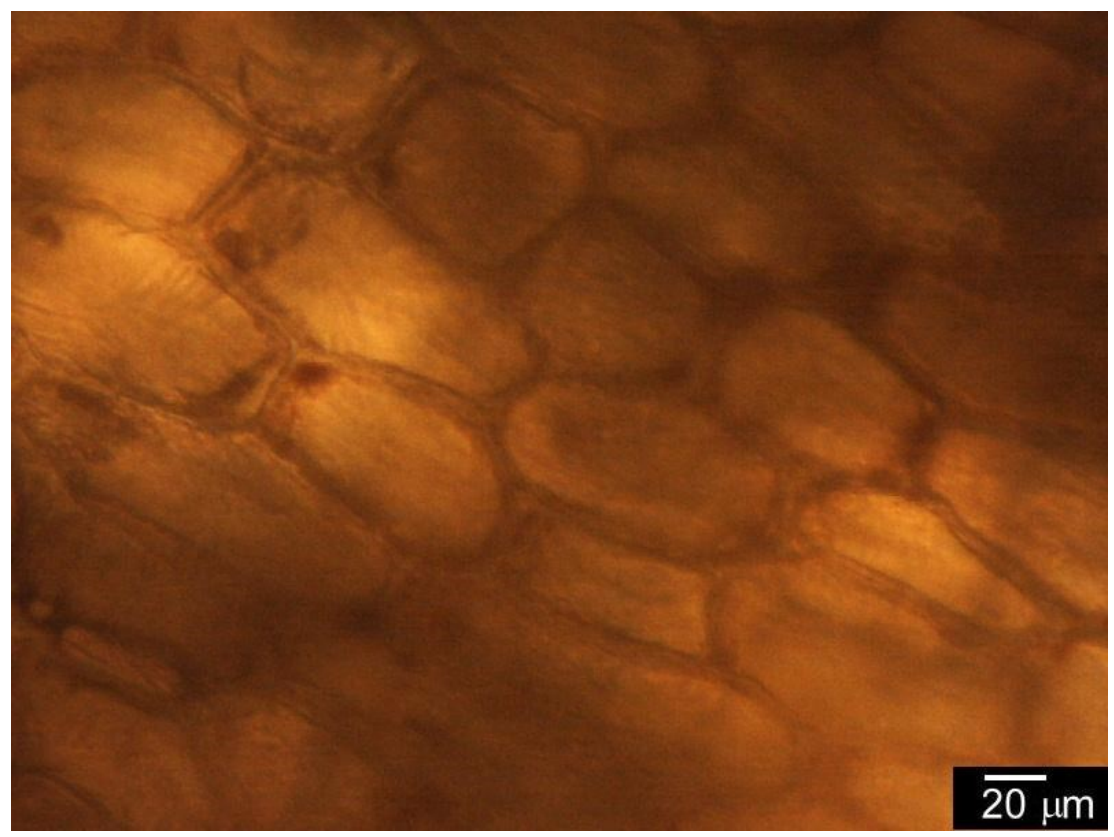

Figure 4. Optical microscopy image of polypyrrole deposited on Japanese camellia.

Fig. 5 (left) shows an optical microscopy image of shell membrane of egg with transmission light. The protein textile structure forms thin membrane inside of the egg shell. Fig. 5 (right) displays the resultant polypyrrole deposited on the membrane of egg with reflection light. This picture indicates polypyrrole forms serpentine configuration, which is a different form from the egg membrane structure. Polymerisation of aniline on the shell membrane is possible as well [9]. 


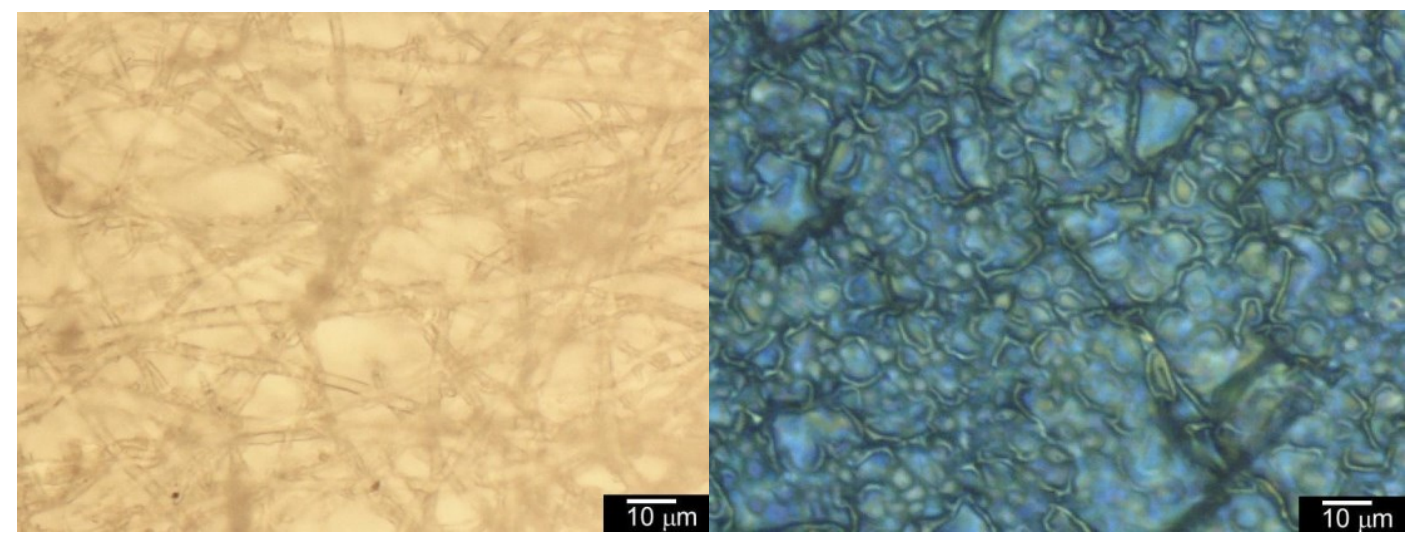

Figure 5. (Left): Optical microscopy image of shell membrane of egg with transmission light. (Right): Optical microscopy image of polypyrrole deposited on shell membrane with reflection light.

Fig. 6 displays an optical microscopy image of electrochemically prepared PEDOT in fermented soybeans bacteria in water solution. The fermented soybeans bacteria on the ITO surface are covered with PEDOT. This surface fine structure is derived from collective of the bacteria.

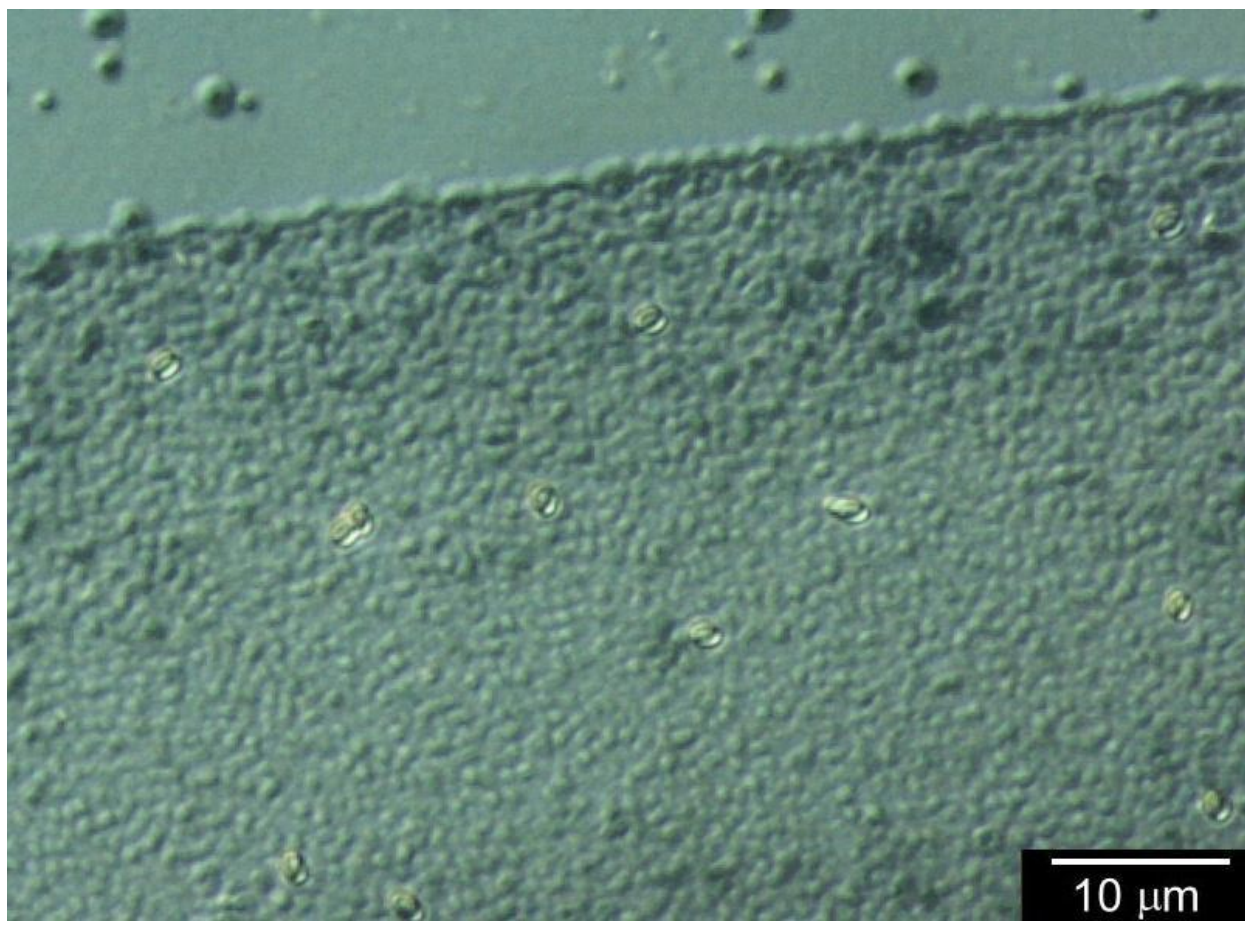

Figure 6. Optical microscopy image of poly(3,4-ethylenedioxythiophene) (PEDOT) prepared in disperse fermented soybeans bacteria/water solution.

\section{Conclusions}

A series of bio-materials/conducting polymer as bio-composites was prepared. Bio-materials can conveniently obtain from natural circumstance. In general, conducting polymers have poor solubility and fusibility. Processing of non-substituted conducting polymers is somewhat difficult. Introduction of flexible side-chain onto the conducting polymers allows improvement of processability. However, the introduction of the side chain results in decrease of electrical conductivity. Therefore, non-substituted conducting polymers are preferable for applications. Combination of functional substrates and non-substituted conducting polymer affords to yield conducting polymer composites having certain surface structure with large size. The method thus performed in this study allows preparation of electro-active composites with micro-size ordered structure with an aid of highly organized structure of bio-tissue. 
This study demonstrated polymerisations, oxidative chemical polymerisation and electrochemical polymerisation, in bacterial solution, and biological tissues to prepare 1) micro-size strings, 2) uniaxially aligned sample along the direction of wood tissue, 3) small chambers (cells) structure, and 4) fine-textile structure on the shell membrane. These ordered composites may be applied for micro-electronics. Note that this study can be a first example of electrochemical polymerisation in bacterial electrolyte solution.

\section{Acknowledgment}

This research was supported by Tsukuba Research Center for Interdisciplinary Materials Science (TIMS).

\section{Materials and Method}

Fermented soybean was commercially obtained in Japan. Shell membrane was carefully removed out of inside of a hen's egg. Chamaecyparis obtuse, camellia, and Japanese cypress were sampled in Tsukuba, Japan. 3,4-Ethylenedioxythiophene (Aldrich, USA) was used as received. Pyrrole (Tokyo Chemical Industry (TCI), Japan) was purified by distillation prior to use. Optical textures were observed using a high-resolution polarising microscope (Nikon ECLIPS LV 100) with a Nikon LU Plan Fluor and Nikon CFIUW lenses.

\section{References}

[1] K. Kawashima, Y. Tamai, H. Ohkita, I. Osaka, K. Takimiya, High-efficiency polymer solar cells with small photon energy loss, Nature Commun. 6 (2015) 10085

[2] K. Saranya, Md. Rameez, A. Subramania, Developments in conducting polymer based counter electrodes for dye-sensitized solar cells - An overview, Euro. Polym. J. 66 (2015) 207-227.

[3] Y. Tao, W. Feng, G. Ding, G. Cheng, Polyaniline nanorods/PVC composites with antistatic properties, Russian J. Phys. Chem. A, 89 (2015) 1445-1448.

[4] F. Laurent M. Sam, M. A. Razali, K.D.G.I. Jayawardena, C.A. Mills, Lynn J. Rozanski, M.J. Beliatis, S.R.P. Silva, Silver grid transparent conducting electrodes for organic light emitting diodes, Org. Electron. 15 (2014) 3492-3500.

[5] Y. Kudo, H. Goto, Bio-interface polymerisation: synthesis of polyaniline on the marine algae surface, Int. Lett. Nat. Sci., 51 (2016) 14-20.

[6] H. Goto, Polymerisation of aniline on the butterfly scale: bio-interface polymerisation Int. Lett. Chem, Phys. Astro., 62 (2015) 34-36.

18052/www.scipress.com/ILCPA.62.34

[7] B. Weng, J. Diao, Q. Xu, Y. Liu, C. Li, A. Ding, J. Chen, Bio-interface of conducting polymer-based materials for neuroregeneration. Adv. Mater. Int. 2 (2015) 1500059-1-1500059-23.

[8] P.A. Calvo, J. Rodríguez, H. Grande, J. Pomposo, Chemical oxidative polymerization of pyrrole in the presence of $m$-hydroxybenzoic acid- and $m$-hydroxycinnamic acid-related compounds, Synth. Met. 126 (2002) 111-116.

[9] Y. Kaitsuka, H. Goto, Synthesis and characterization of polyaniline composite with shell membrane, Fib. Polym., 17 (2016) 815-819. 